\title{
Percutaneous Mastoid Electrical Stimulator improves poststroke depression and cognitive function in patients with ischemic stroke:A Prospective, Randomized, Double-blind, and Sham-controlled Study
}

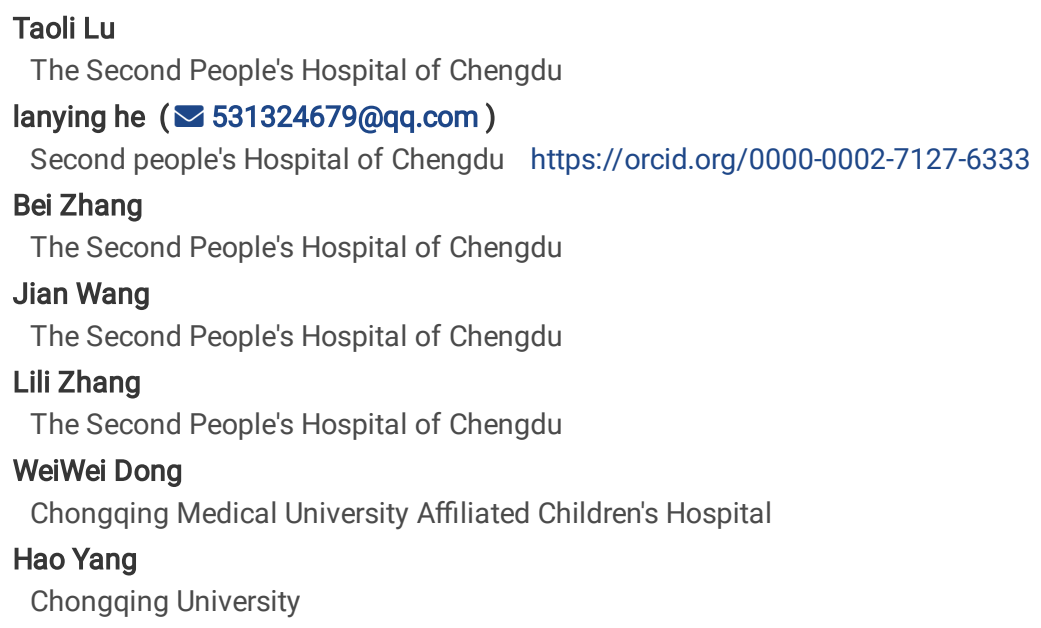




\section{Abstract}

Backgrond : Poststroke depression could lead to functional dependence, cognitive impairment and reduced quality of life. The aim of this study was to evaluate the effects of percutaneous mastoid electrical stimulator (PMES) plus antidepressant on poststroke depression and cognitive function. Methods: This study was a prospective, randomized, double-blind, and sham-controlled study . 258 clinically depressed ischemic stroke patients within $14 \mathrm{~d}$ of index stroke were randomly assigned to PMES plus antidepressant (PMES group, $\mathrm{N}=125$ ) and sham plus antidepressant (sham group, $\mathrm{N}=133$ ). All patients underwent Montreal Cognitive Assessment (MoCA) and Hamilton Rating Scale for Depression (HRSD) test at 2 weeks (baseline), and 6 months after the stroke. Primary outcomes were the percentage of treatment response ( $\geq 50 \%$ reduction in HRSD score) and depression remission (HRSD score $\leq 9)$ at 6 months. Secondary outcome was the percentage of MoCA score <26. Results: The percentage of treatment response and depression remission in PMES group were significantly higher than that in the sham group ( $57.60 \%$ vs $41.35 \%, P=0.009)$, (44.00\% vs $29.32 \%, P=0.014)$, respectively. The mean value of HRSD score change(M6-baseline) was significantly greater in the PMES group compared to sham group at 6 months $(-11.93 \pm 5.32$ vs $-10.48 \pm 6.10, P=0.036)$. The percentage MoCA score $<26$ in PEMS group was lower than that in sham group $(12.0 \%$ vs $24.06 \%$, $\mathrm{P}=0.012$ ), the mean value of MoCA score change (M6-baseline) was higher in PMES group compared to sham group ( $3.50 \pm 2.55$ vs $2.72 \pm 2.52$; $\mathrm{P}=0.005$ ). Conclusion: These findings demonstrate that PMES adjunctive to antidepressant therapy is effective in reducing depression, achieving remission in the short term, and improving cognition. Trial registration: This trial was retrospectively registered (registration number: ChiCTR1800016463) on 03 June 2018

\section{Background}

Stroke is a leading cause of long-term disability. Despite of impressive progress in early diagnosis and medical treatment which result in the decrease of incidence and mortality rates of stroke, about $25 \%-74 \%$ stroke patients still suffer major disability and psychological illness, including depression, cognitive impairment, and social isolation[1,2]. Poststroke depression (PSD) is associated with poor outcomes after stroke, including cognitive disorders, poor rehabilitation outcomes[3,4]. PSD has a prevalence of about $30 \%$ in stroke survivors based on the previous studies[5].

The treatment of PSD includes medication and psychotherapy[6-11]. Selective serotonin reuptake inhibitors (SSRIs) are the most used drugs in the treatment of PSD[6-8]. However, some patients are reported to experience insufficient efficacy and adverse events. Psychotherapy has poor effect on PSD. Hence, it is very important to find a non-pharmacologic treatment for PSD $[10,11]$.

In 1998, neuroprotection of fastigial nucleus stimulation (FNS) was first confirmed by Reis at al [12], the result showed that one hour of FNS treatment in anesthetized rats prior to middle cerebral artery occluded (MACO) reduced the volume of the focal infarction to $50 \%$. In recent decades, many studies have shown that FNS has a variety of neuroprotective mechanisms [13]. It can inhibit the electrical activity around the focus, reduce the excitotoxic injury of neurons, inhibit the inflammatory response, and inhibit apoptosis [13].

Non-invasive percutaneous mastoid electrical stimulator (PMES) is called cerebrovascular function therapy (CVFT) device in China, using a biological bionic current to therapeutically stimulate fastigial nucleus (FN), it is proved by animal experiments that FN stimulation can be achieved extracranially [14].During electrical stimulation, excited nerve fibers pass through FN, resulting in increased blood pressure, reflexive vasodilatation and increased cerebral blood flow(CBF), which are called the fastigial pressor response (FPR) [15]. By inhibiting baroreceptor reflex, FRR is enhanced, adrenaline, noradrenaline and arginine vasopressin were released [16,17]. The increase of CBF is global (including spinal cord), and the largest increase is in frontal lobe and parasaggital area of cortex $[18,19]$. Fastigial nucleus stimulation can induce neuroprotection against cerebral ischemia囚electrical stimulation of cerebellar dentate nucleus or white matter does not have a neuroprotective effect. In addition, FNS treatment after selective injury of FN neurons failed to induce neuroprotection, suggesting that the protection of FNS to cerebral ischemia was generated in the intrinsic FN neurons [20].

FNS has been reported to improve depression and cognitive function after stroke in animal experiments [21-23]. Some observation studies show that PMES treatment can improve the clinical prognosis and show good safety [24-27]. However, due to the small number of cases in these studies, the clinical efficacy of FNS in PSD still lacks enough evidence. The purpose of this study was to explore the effect of PMES combined with antidepressants on PSD and cognitive function.

\section{Methods}

\section{Study Population}

This study was a prospective, randomized, double-blind, and sham-controlled study. This project was registered in the Chinese Clinical Trial Register (ChiCTR) (Registration for trial number ChiCTR1800016463 was retrospectively completed on June 3,2018) and was performed according to the CONSORT 2010 extension to randomized pilot and feasibility trials [28]. The patients were admitted to the Second People's Hospital of Chengdu due to ischemic stroke within 14 days of symptom onset between January 2015 and December 2018. Ischemic stroke was confirmed by brain computed tomography or magnetic resonance imaging.

\section{Depression Screening}

Patients screened positive for depressive symptoms, and whose diagnosis of clinical depression was verified by a diagnostic interview using DSM V criteria. Depression screening was carried out by 30-item Geriatric Depression Scale (GDS),which consists of 30 questions, are scored as 1 point 
individually, resulting in 0-30 points and being classified as: 0-10, no depression; 11-20, mild depression; 21-30, moderate depression. The diagnosis of depression was validated by Hamilton Rating Scale for Depression (HRSD) in those who scored $\geq 11$ on the GDS and consented to the full study. Stroke severity was assessed based on National Institutes of Health Stroke Scale (NIHSS). The study was approved by the ethics committees of the Second People's Hospital of Chengdu. Informed consent was signed by all the participants.

\section{Inclusion and exclusion criteria}

Patients were included if they fulfilled all the following criteria: (1) admission for first-ever ischemic stroke within 14 days, (2) no neurological or psychiatric disease before stroke, (3) no aphasia,(4) no drug abuse, (5) severe hearing deficit unable to complete the scale, (6) right-handed, (7) no serious dysarthria and (8) able to co-operate,(9) no active malignancies;(10) patients could appropriately communicate.

\section{Study Design and grouping}

Patients were divided into two groups: the sham and PMES groups. The patients in PMES group received PMES treatment as an add-on to antidepressant and the patients in sham group received sham stimulation and antidepressant.

\section{Treatment methods}

After cleaning the bilateral mastoid skin behind the ears, stimulation electrodes were placed. The electrode size was $42 \times 24 \mathrm{~mm}$ and the conductive area was $19 \mathrm{~mm}$ (Figure 1). The stimulus parameters were set as follows: pulse width $90 \mathrm{mS}$ for both PMES and sham, frequency $1.8 \mathrm{kHz}$ for PMES and 10 $\mathrm{Hz}$ for sham, peak current 10mA for PMES and 0.18mA for sham [29]. Previous studies have shown that $10 \mathrm{~mA}$ is very safe, and some patients may have slight tingling, but no skin redness or burn [29]. In order to reduce the surface sensation caused by current stimulation, we modulated the lowfrequency signal $(13-45 \mathrm{hz})$ to the intermediate frequency signal of $1.8 \mathrm{kHz}$ and set $1.0-1.2 \mathrm{v}$ as the voltage range of the low-frequency signal. This change in the modulation signal in this range causes a slight squeeze. The intermediate-frequency signal was the exponential decay signal with a base of "a" $(0<a<1)$. The signal was a non-polar exponential waveform, which was composed of positive and negative pulse waves and equivalent charges. Negative pulse can depolarize the nerve fiber, while positive pulse can balance the charge, thus eliminating the accumulation of electrostatic charge and reducing the adverse electrochemical reaction. In order to reduce the energy of single pulse, we reduce the base value "a", not the pulse width, thus reducing the degree of extrusion. The surface sensation of real stimulus was close to the surface sensation of sham stimulus, which was a periodic point-contact sense of touch. PMES group and sham group were treated 45 minutes/day lasted 6 months.

In this study, selective serotonin reuptake inhibitor (SSRI) was recommended as the first choice for depressive patients, and sertraline was recommended as the initial antidepressant because of its tolerance to medical treatment and relatively low incidence of cardiovascular side effects. The patients were prescribed sertraline $50 \mathrm{mg} /$ day, the dose was adjusted starting from day 7 into $100 \mathrm{mg} /$ day (maximum dose $400 \mathrm{mg} /$ day). If patients could not tolerate the side effects of sertraline, another antidepressant was prescribed (escitalopram or paroxetine).

\section{Randomization and double blinding}

Patients who met the criteria were assigned to treatment groups according to a predefined randomization plan by using a block size of 4 , a ratio of $1: 1$, and stratified by study team. A computer-generated block randomization list has been prepared by the Clinical Research Unit of The Second People's Hospital of Chengdu. The randomization was conducted by a statistical analyzer who was not involved in other parts of the study. Patients, investigators and all study personnel were blinded to the treatment allocation. The PMES and sham stimulators had the same external appearances, user manuals and electrodes. They could not be distinguished by their external appearance. We took the following measures to guarantee doubleblinding: enrolled patients were not acquainted with each other, there was no physical contact or communication (such as sensory perception) between patients during visits, and all of the patients would be told when enrolled that it was not possible to accurately judge whether they were receiving true or sham stimulation only based on the surface sensation.

\section{Data Collection}

Baseline characteristics including demographics, stroke characteristics, NIHSS, risk factors. All patients underwent depressive state and cognitive assessment at 2 weeks (baseline) and 6 months after ischemic stroke.

Depressive state was assessed using HRSD score. Treatment response was defined as $\geq 50 \%$ reduction in HRSD score. Remission has been defined variably as HRSD score of $\leq 9$ (no longer meeting depression criterion), $\leq 7$ (absence of any depressive symptoms), or $\leq 3$ (equivalent to healthy controls). We used HRSD score of $\leq 9$ and $\geq 50 \%$ reduction in HRSD score for comparison with baseline. Cognitive state was assessed using the Montreal Cognitive Assessment (MoCA), scores range from 0 to 30 points, with a lower score reflecting greater cognitive impairment, and a cut-off of $<26$ was considered as indicative of cognitive impairment.

All patients were followed up for 6 months. After discharge, the patients completed treatment at home or nursing home. The patients or caregivers in both groups were trained in using the PMES and sham. All patients were followed up once a month by face-to-face interview or telephone interview.

The change in HRSD and MoCA score was detected at 6 months after treatment. Primary outcomes were treatment response ( $\geq 50 \%$ reduction in HRSD score) and depression remission (HRSD score $\leq 9)$ at 6 months after ischemic stroke. Secondary outcome was the percentage of 6-month MoCA score 


\section{Statistical analysis}

The treat response rate of the PMES group and sham group were about $55 \%$ and $35 \%$, respectively. To examine the significant difference between these two groups, the bilateral significance level was established at $5 \%$, and the power of the test was $80 \%$. Considering a $20 \%$ loss to follow-up, the sample size of each group was estimated at approximately 120 cases.

Demographic characteristics and vascular risk factors were compared between sham and PMES groups. Continuous data were expressed as mean values ( \pm standard deviation), using the Mann-Whiney U test. Categorical data were described using frequency and percentage, using Pearson $\chi 2$ test, Fisher exact 2-sided test. The data were analyzed using SPSS software (SPASS 22.0). P values<0.05 were considered as statistically significant.

\section{Result}

\section{Characteristics of the study subjects}

About 1000 patients with ischemic stroke were tracked for potential screening eligibility. Some patients were not eligible(aphasia $₫$ severe hearing deficit $\$ psychiatric disease before stroke冈drug abuse). 810 patients agreed to be screened. 305 patients were found eligible (GDS $\geq 11$ ), 17 patients refused; 288 patients enrolled. (See Figure 2 for details on exclusions).

A total of 288 patients were enrolled in this study (sham group, $\mathrm{N}=144$; PMES group, $\mathrm{N}=144$ ). 12 patients were lost to follow-up after discharge from the hospital (sham group, $\mathrm{N}=3$; PMES group, $\mathrm{N}=9$ ), recurrent stroke occurred in 10 patients(sham group, $\mathrm{N}=4$; PMES group, $\mathrm{N}=6$ ), 8 patients had died during the 6-month follow-up period(sham group, N=4; PMES group, N=4). 258 patients were finally analyzed (sham group, N=133; $P M E S$ group, $N=125$ ) (Figure 1), comprised $52.33 \%$ (135) men and $47.67 \%$ (123) women, the mean age was $65.58 \pm 8.59$ years (range:42-87 years). In the study population, 148 patients had a history of hypertension, 97 had a history of diabetes, 139 had a history of hyperlipidemia, 91 patients smoke. The PMES and sham groups received treatment daily for 45 minutes, and the treatment lasted 6 months. There were no adverse reactions reported either in the PMES group or in the sham group during the treatment period.

Baseline characteristics of patients in the sham group and the PMES group were compared (Table1). Sertraline, escitalopram and paroxetine were the most commonly prescribed SSRI drugs. No patients stopped taking antidepressant during the follow-up period. There were also no significant group differences in the baseline HRSD and MoCA score (Pख0.05).

\section{Primary outcomes}

There was no difference in HRSD score at baseline in sham and PEMS groups (22.02 \pm 4.54 vs $21.51 \pm 4.32 ; P=0.280)(T a b l e ~ 1)$. At the end of the 6-month intervention period, HRSD score improved both in sham and PMES groups(Table 2a), HRSD score was lower in PEMS than that in the sham group $(9.58 \pm 3.45$ vs $11.54 \pm 4.21, P<0.001)$, the mean value of HRSD score change(M6-baseline) was significantly greater in the PMES group compared to sham group at 6 months $(-11.93 \pm 5.32$ vs $-10.48 \pm 6.10, P=0.036)$ (Table 3$)$.

During the 6-month follow-up period, 126 patients had treatment response, 94 patients had depression remission (Table $2 \mathrm{~b}$ ). Treatment response in sham group were $41.35 \%(55 / 133)$ at 6 months, which was significantly lower than that in the PMES group $(57.60 \%, 72 / 125)(P=0.009)$.Depression remission in sham group were $29.32 \%(39 / 133)$ at 6 months, which was significantly lower than that in the $\mathrm{PMES}$ group $(44.00 \%, 55 / 125)(P=0.014)$.

\section{Secondary outcomes}

At baseline, there was no difference in MOCA score in sham and PEMS groups(24.90 \pm 2.82 vs $24.89 \pm 3.16 ; P=0.936)($ Table 1$)$, the percentage of MoCA score $<26$ was not different between PEMS and sham groups[57.60\%(72/125) vs54.89\%(73/133),P=0.661]. At the end of the six-month intervention period, MoCA score improved both in sham and PMES groups, the percentage MoCA score <26 in PEMS group was lower than that in sham group[12.00\%(15/125) vs 24.06\%(32/133), P=0.012], MoCA score in PMES group was higher than that in sham group at 6 month (28.26 \pm 1.95 vs $27.26 \pm 2.20, \mathrm{P}<0.001$ ), in addition, the mean value of MoCA score change (M6-baseline) was higher in PMES group(3.50 \pm 2.55$)$ compared to sham group $(2.72 \pm 2.52 ; \mathrm{P}=0.005)$ (Table 3).

\section{Adverse reactions and compliance}

There were no adverse reactions reported either in the PMES group or in the sham group during the treatment period. The mean number of applications of the device over the 6 months was 166 (92.22\%) in the PMES group and 159 (88.33\%) in the sham group. The difference between the two groups was not significant $(P=0.213)$.

\section{Discussion}

The primary and secondary outcome of this randomized, sham-controlled study showed that daily treatment with PMES in combination with pharmacotherapy was more effective in PSD. The results of the study showed that PMES and sham treatment were both effective in improving PSD and cognition. At the end of the 6-month follow-up period, compared with PMES, the drop in HRSD score, the percentage in treatment response and 
depression remission were smaller in sham group. In addition to the improvement in PSD, the secondary outcome, MoCA score also showed a significant increase in two group. the increased in MoCA score was lower and the percentage of MoCA score $₫ 26$ was higher in sham group compared to PMES group at 6 months.

The incidence of PSD is very high. PSD affects $12 \%-72 \%$ of stroke patients [30,31]. A meta-analysis showed that $31 \%$ of patients developed depression within 5 years after stroke. In the past, physical disability caused by stroke was often the focus of treatment. However, in recent years, the treatment of psychological comorbidities had also attracted the attention of clinicians, which affected the effect of patients' rehabilitation. After stroke, much of patients suffered motor impairment, which limit their mobility, lost confidence, which may lead to PSD [32]. The previous studies had proven the positive effects of PEMS on motor function[25,26],animal experiments showed that FNS alone or in combination with drug therapy could improve PSD [33],while in clinical practice, the effect of PMES on PSD was unclear. Hence, in the present study, we investigated the effect of PMES on PSD assessed by HRDS, and we found that PMES combined with antidepressant had been significantly more successful in improving poststroke depression than medication alone. In this study, during the 6-month follow-up period, the higher percentage of patients having a HRSD score of $\leq 9$ and $\geq 50 \%$ reduction in the PMES group compared to the sham group showed that more patients from the PMES group regained less depressed. The effects of sham stimulation in this study might involve antidepressants during the treatment period. From every outcome measure, the preventive effect of the PMES treatment was much better than sham treatment. Therefore, the improved effect of PMES treatment in PSD mainly derived from the PMES treatment itself.

Cognitive impairment that is one of common sequelae after stroke. Cerebellum plays a role in cognition [34-35]. Stroke can affect cerebellar function and produce vascular dementia (VD). The study had found that activation of the cerebellum significantly alleviated VD, and poststroke cognitive impairment was improved by FNS treatment [17]. Fan et al. found that the cognitive function decreased on 2 months after chronic cerebral hypoperfusion, and was worse on 4 months after hypoperfusion, the cognitive function improved after FNS treatment [17]. Although animal study had shown that PMES could improve cognitive function after cerebral ischemia, limited information about the role of PMES in cognition impairment after stroke in clinical study. In our study, we observed that PMES could improve cognition in ischemic stroke patients, the mean value of MoCA score change was higher in PMES group compared to sham group, and the percentage MoCA score <26 in PEMS group was lower than that in sham group.

The exact mechanism of action of PMES is unclear. According to previous studies, FNS could up-regulate NE and 5-HT in the frontal lobes of rats with depression[36,37], in additional, the positive affective state or enhanced arousal and attention could improve cognition, which seems a plausible mechanism[38].

Some limitations of this study merit consideration. Firstly, NIHSS score has been shown to correlate with infarction volume, we lacked data on infarction volume. Secondly, peak current is $10 \mathrm{~mA}$ for PMES and $0.18 \mathrm{~mA}$ for sham, which might give patients clue about group assignment and have an effect on the experimental results. Thirdly, each group were prescribed and reported taking antidepressants during the 6 months treatment period, the doses and type of drug were not standardized. In addition, cognitive status was assessed using MoCA, but this questionnaire will also be affected by education level, the cutoff was not adjusted for people with low literacy, which may have some deviation to the results. This is a limitation of the study but represents the context of everyday practice.

\section{Conclusions}

In conclusion, our findings indicated that PMES adjunctive to antidepressant therapy is effective in reducing depression and achieving remission in the short term. We also demonstrated that improved poststroke depressive was associated with improved cognition. These data indicate that PMES may be a safe and low-cost therapy to improve clinical stroke outcomes.

\section{Abbreviations}

percutaneous mastoid electrical stimulator (PMES); Hamilton Rating Scale for Depression (HRSD); Montreal Cognitive Assessment (MoCA); Poststroke depression (PSD); Fastigial nucleus stimulation (FNS); cerebrovascular function therapy (CVFT); National Institutes of Health Stroke Scale(NIHSS)

\section{Declarations}

\section{Acknowledgments}

The devices were provided by Chongqing Haikun Medical Instrument Co., Ltd. None of the investigators has any financial interest in Chongqing Haikun Medical Instrument Co., Ltd. We thank all patients and their families for generously consenting to use the data in this research.

\section{Authors' contributions}

TLL was responsible for the data collection and analysis and the first draft of the paper and further manuscript. LYH was responsible for the concept and design of the study.BZ was responsible for the data collection and analysis. JW was responsible for the design of the study.LLZ was responsible for the data analysis, and interpretation. WWD was responsible for the interpretation. HY was responsible for the data analysis All authors read and approved the final manuscript for publication.

\section{Funding}


This work was funded by the Health and Family Planning Commission of Chengdu (2015009). The funding body did not participate in designing the study or writing the manuscript. The study protocol has undergone peer-review process by the funding body.

\section{Availability of data and materials}

Data used in this study may be available by request to corresponding author via email: 531324679@qq.com

\section{Ethics approval and consent to participate}

We obtained ethical approval for this study from the Medical and Health Research Ethics Committee at Second people's Hospital of Chengdu. The current study was carried out according to Declaration of Helsinki. Written informed consent was obtained from all study participants or their legal proxies.

\section{Consent for publication}

Not applicable.

\section{Competing interests}

The authors declare that they have no competing interests.

\section{Reference}

1. Stein LA, Goldmann E, Zamzam A, Luciano JM, Messé SR, Cucchiara BL, Kasner SE, Mullen MT.Association Between Anxiety, Depression, and Post-traumatic Stress Disorder and Outcomes After Ischemic Stroke. Front Neurol.2018;9:890. https://doi: 10.3389/fneur.2018.00890. eCollection 2018

2. Towfighi A, Ovbiagele B, El Husseini N, Hackett ML, Jorge RE, Kissela BM, Mitchell PH, Skolarus LE, Whooley MA, Williams LS; American Heart Association Stroke Council; Council on Cardiovascular and Stroke Nursing; and Council on Quality of Care and Outcomes Research. Poststroke Depression:A Scientific Statement for Healthcare Professionals From the American Heart Association/American Stroke Association.Stroke.2017;48(2):e30-e43. https://doi: 10.1161/STR.0000000000000113.

3. Love MF, Sharrief A, Chaoul A, Savitz S, Beauchamp JES. Mind-Body Interventions, Psychological Stressors, and Quality of Life in Stroke Survivors.Stroke. 2019;50(2):434-440. https://doi: 10.1161/STROKEAHA.118.021150

4. Huang J, Zhou FC, Guan B, Zhang N, Wang A, Yu P, Zhou L, Wang CY, Wang C. Predictors of Remission of Early-Onset Poststroke Depression and the Interaction Between Depression and Cognition During Follow-Up. Front Psychiatry. 2019;9:738. https://doi:10.3389/fpsyt.2018.00738. eCollection 2018.

5. De RA, Fransen E, Brouns R, Geurden M, Peij D, Mariën P, De Deyn PP, Engelborghs S.Poststroke depression and its multifactorial nature: results from a prospective longitudinal study. J Neurol Sci.2014;347(1-2):159-166. https://doi:10.1016/j.jns.2014.09.038.

6. Choi-Kwon S, Han SW, Kwon SU, Kang DW, Choi JM, Kim JS. Fluoxetine treatment in poststroke depression, emotional incontinence, and anger proneness: a double-blind, placebo-controlled study. Stroke.2006;37(1):156-161. https://doi:10.1161/01.STR.0000190892.93663.e2.

7. FOCUS Trial Collaboration. Effects of fluoxetine on functional outcomes after acute stroke (FOCUS): a pragmatic, double-blind, randomised, controlled trial. Lancet. 2019;393(10168):265-274. https://doi:10.1016/S0140-6736(18)32823-X.

8. Villa RF1, Ferrari F2, Moretti A2.Post-stroke depression: Mechanisms and pharmacological treatment. Pharmacol Ther. 2018;184:131-144. https://doi:10.1016/j.pharmthera.2017.11.005.

9. Karaiskos D, Tzavellas E, Spengos K, Vassilopoulou S, Paparrigopoulos T. Duloxetine versus citalopram and sertraline in the treatment of poststroke depression, anxiety, and fatigue. J Neuropsychiatry Clin Neurosci. 2012;24(3):349-353. doi: 10.1176/appi.neuropsych.11110325

10. Kohen R, Cain KC, Buzaitis A, Johnson V, Becker KJ, Teri L, Tirschwell DL, Veith RC, Mitchell PH.Response to psychosocial treatment in poststroke depression is associated with serotonin transporter polymorphisms. Stroke.2011;42(7):2068-2070. https://doi:10.1161/strokeaha.110.611434.

11. Mitchell PH, Veith RC, Becker KJ, Buzaitis A, Cain KC, Fruin M, Tirschwell D, Teri L. Brief psychosocial-behavioral intervention with antidepressant reduces poststroke depression significantly more than usual care with antidepressant: living well with stroke: randomized, controlled trial. Stroke 2009;40(9):3073-3078. https://doi: 10.1161/STROKEAHA.109.549808

12. Reis DJ, Kobylarz K, Yamamoto S, Golanov EV. Brief electrical stimulation of cerebellar fastigial nucleus conditions long-lasting salvage from focal cerebral ischemia: Conditioned central neurogenic neuroprotection. Brain Res. 1998; 780(1): 161-165[PMID:9497093]

13. Wang J, Dong WW, Zhang WH, Zheng J, Wang X.

Electrical stimulation of cerebellar fastigial nucleus: mechanism of neuroprotection and prospects for clinical application against cerebral ischemia. CNS. Neurosci Ther. 2014;20(8):710-716. https://doi:10.1111/cns.12288.

14. Xia YL, Luo Y, Dong WW. Effect and mechanism of fastigial nucleus stimulation on stroke in rats. J Apoplexy Nerv Dis. 1999;16:3-5.

15. Miura M, Reis DJ. Cerebellum: A pressor response elicited from the fastigial nucleus and its efferent pathway in brainstem. Brain Res 1969;13(3):595-599. https://doi:10.1016/0006-8993(69)90269-8. 
16. Del Bo A, Sved AF, Reis DJ. Fastigial stimulation releases vasopressin in amounts that elevate arterial pressure. Am J Physiol.1983;244:H687H694.

17. Del Bo A, Sved AF, Reis DJ. Inhibitory influences from arterial baroreceptors on vasopressin release elicited by fastigial stimulation in rats. Circ Res.1984;54(3):248-253. https://doi:10.1161/01.res.54.3.248.

18. Golanov EV, Reis DJ. Cerebral cortical neurons with activity linked to central neurogenic spontaneous and evoked elevations in cerebral blood flow. Neurosci Lett.1996;209(2):101-104. https://doi:10.1016/0304-3940(96)12611-2.

19. Nakai M, ladecola C, Reis DJ. Global cerebral vasodilation by stimulation of rat fastigial cerebellar nucleus. Am J Physiol.1982;243(2):H226-H235. https://doi:10.1152/ajpheart.1982.243.2.H226.

20. Glickstein SB, Golanov EV, Reis DJ. Intrinsic neurons of fastigial nucleus mediate neurogenic neuroprotection against excitotoxic and ischemic neuronal injury in rat. J Neurosci.1999;19(10):4142-4154.[ PMID:10234042]

21. Sui R, Zhang L, Min L, Yuan J, Li X. Cerebellar dysfunction may play an important role in post-stroke depression. Med Hypotheses.2009;72(6):643646. https://doi:10.1016/j.mehy.2008.11.042

22. Baldacara L, Borgio JG, Lacerda AL, Jackowski AP. Cerebellum and psychiatric disorders. Rev Bras Psiquiatr.2008;30(3):281-289. https://doi:10.1590/s1516-44462008000300016

23. Fan XL, Dong WW, Yang QD. The prophylactic and curative effects of electrical stimulation of cerebellar fastigial nucleus on vascular dementia in rats. Stroke Nerv Dis.2004;11:349-352.

24. Niu LC, Li T, Lei JA, Han Q, Li YX, Zhang BQ, Yan PJ. Effect of cerebellar fastigial nucleus treated by electrical stimulation on 70 patiengts with acute cerebral infarction. Chin J Rehabil Theory Practice.2005;11:795-796.

25. Xu X. Application of cerebellar fustigial nucleus electric stimulation on rehabilitation of cerebrovascular diseases. Lab Med Clin,2010;7:1318-1321.

26. Shen HQ. Cerebral circulation function therapeutic apparatus of the role of the mechanism and clinical effect. Prog Biomed Eng.2009;30:245-246.

27. He WY, Li BP, Huang XZ. Clinical observation of treatment of acute cerebral infarction by electrical stimulation of the fastigial nucleus. J Apoplexy Nerv Dis.2003;20:470.

28. Eldridge SM, Chan CL, Campbell MJ, Bond CM, Hopewell S, Thabane L,Lancaster GA. PAFS consensus group: CONSORT 2010 statement: extension to randomised pilot and feasibility trials. BMJ. 2016;355:i5239. https://doi: 10.1136/bmj.i5239

29. Juan Y, Shu O, Jinhe L, Na Y, Yushuang D, Weiwei D, Lanying H, Jian W. Migraine prevention with percutaneous mastoid electrical stimulator: A randomized double-blind controlled trial. Cephalalgia.2017;37(13):1248-1256. https://doi: 10.1177/0333102416678623

30. Paolucci S. Epidemiology and treatment of post-stroke depression. Neuropsychiatr Dis Treat.2008;4(1):145-117. https://doi: 10.2147/ndt.s2017

31. Torrisi M, De Cola MC, Buda A, Carioti L, Scaltrito MV, Bramanti P, Manuli A, De Luca R, Calabrò RS. Self-Efficacy, Poststroke Depression, and Rehabilitation Outcomes: Is There a Correlation?J Stroke Cerebrovasc Dis. 2018;27(11):3208-3211. https://doi:10.1016/j.jstrokecerebrovasdis.2018.07.021.

32. Ghaffari A, Akbarfahimi M, Rostami HR. Discriminative factors for post-stroke depression. Asian Journal of Psychiatry. $2019,48: 101863$. https://doi:10.1016/j.ajp.2019.101863. https://doi:10.1016/j.ajp.2019.101863

33. Zhang L, Zhao M, Sui RB.Cerebellar Fastigial Nucleus Electrical Stimulation Alleviates Depressive-Like Behaviors in Post-Stroke Depression Rat Model and Potential Mechanisms.Cell Physiol Biochem. 2017;41(4):1403-1412. https://doi:10.1159/000467940.

34. Schmahman JD, Sherman JC. The cerebellar cognitive affective syndrome. Brain 1998;121(Pt4):561-579. https://doi:10.1093/brain/121.11.2202

35. Dolan RJ, Bench CJ, Brown RG, Scott LC, Friston KJ. Frackowiak RS. Regional cerebral blood flow abnormalities in depressed patients with cognitive. J Neurol Neurosurg Psychiatry.1992;55(9):768-773. https://doi:10.1136/jnnp.55.9.768.

36. Gao W, Wang N, Qiao H.The effects of 5-HT1B receptor subtypes on motor behaviors mediated by cerebellar fastigial nucleus. Zhongguo Ying Yong Sheng Li Xue Za Zhi. 2016;32(6):550-554. https://doi:10.13459/j.cnki.cjap.2016.06.014

37. Zhang Runfeng,Li Xia,Chen Yun zhen, Tang Ke xin, Ma Guo-zhong, Huang Xuan yin. Effects of cerebellar fastigial nucleus electro-stimulation on release of neurotransmitters in ischemic hearts. Chinese Journal of Clinical Rehabilitation;9:34-37.

38. Kimura M1, Robinson RG, Kosier JT.Treatment of cognitive impairment after poststroke depression : a double-blind treatment trial. Stroke. 2000;31(7):1482-1486. https://doi:10.1161/01.str.31.7.1482

\section{Tables}

Table 1 Comparison of baseline characteristics at admission between patients with Sham and FNS groups. 


\begin{tabular}{|c|c|c|c|c|}
\hline & Sham group (133) & PMES group (125) & OR(95\%CI) & P* \\
\hline Age, y(Mean SD) & $66.11 \pm 8.37$ & $65.0 \pm 8.82$ & & 0.622 \\
\hline NIHSS, (Mean SD) & $6.99 \pm 2.47$ & $7.02 \pm 2.21$ & & 0.978 \\
\hline Females, n(\%) & $68(51.13)$ & $55(44.00)$ & $0.751(0.46-1.23)$ & 0.465 \\
\hline Men, n(\%) & $65(48.87)$ & $70(56.00)$ & $0.751(0.46-1.23)$ & 0.252 \\
\hline $\mathrm{BMI} \geq 24$ kg/m, n(\%) & $32(24.06)$ & $42(33.60)$ & $1.60(0.93-2.75)$ & 0.090 \\
\hline Hypertension, n(\%) & $72(54.14)$ & $76(60.8)$ & $1.31(0.80-2.16)$ & 0.279 \\
\hline Current Smoking, n(\%) & $48(36.09)$ & $43(34.40)$ & $0.93(0.56-1.55)$ & 0.776 \\
\hline Current Drinking, $\mathrm{n}(\%)$ & $43(32.33)$ & $44(33.08)$ & $1.14(0.68-1.91)$ & 0.626 \\
\hline Diabetes, n(\%) & $54 \llbracket 40.60 \square$ & $43(34.40)$ & $0.77(0.46-1.27)$ & 0.304 \\
\hline Hyperlipidemia, n(\%) & $65(48.87)$ & $74(59.20)$ & $1.52(0.93-2.45)$ & 0.096 \\
\hline Atrial fibrillation, $\mathrm{n}(\%)$ & $50(37.59)$ & $40(30.08)$ & $0.78(0.47-1.31)$ & 0.346 \\
\hline Family history of stroke, $\mathrm{n}(\%)$ & $29(21.80)$ & $33(26.40)$ & $1.29(0.73-2.28)$ & 0.388 \\
\hline MoCA Score, (mean SD) & $24.90 \pm 3.16$ & $24.90 \pm 2.82$ & & 0.936 \\
\hline HRSD Score, (mean SD) & $22.02 \pm 4.54$ & $21.51 \pm 4.32$ & & 0.280 \\
\hline \multicolumn{5}{|l|}{ Medications use } \\
\hline Antiplatelet, n(\%) & $43(32.33)$ & $48(38.40)$ & $1.31 \square 0.78-2.17 \square$ & 0.308 \\
\hline Antihypertensive, n(\%) & $56(42.11)$ & $56(44.80)$ & $1.12(0.68-1.83)$ & 0.663 \\
\hline lipid-lowering medications, n(\%) & $64(48.12)$ & $71(56.80)$ & $1.42 \llbracket 0.87-2.32 \square$ & 0.163 \\
\hline Sertraline, n(\%) & $83(62.41)$ & $81(60.90)$ & $1.11(0.67-1.84)$ & 0.690 \\
\hline Escitalopram, n(\%) & $14(10.53)$ & $19(14.29)$ & $1.52(0.73-3.19)$ & 0.261 \\
\hline $\begin{array}{l}\text { Paroxetine, } \mathrm{n}(\%) \\
\text { Infarct location }\end{array}$ & $36(27.07)$ & $25(18.80)$ & $0.67(0.38-1.21)$ & 0.182 \\
\hline Basal ganglia, n(\%) & $62(46.62)$ & $61(45.86)$ & $1.09(0.67-1.78)$ & 0.726 \\
\hline Brain stem, n(\%) & $18(13.53)$ & $20(15.04)$ & $1.22(0.611-2.43)$ & 0.576 \\
\hline Cerebellum, n(\%) & $10(7.52)$ & $4(3.01)$ & $0.41(0.12-1.33)$ & 0.126 \\
\hline Frontal lobe, $\mathrm{n}(\%)$ & 19(14.29) & $15(11.28)$ & $0.82(0.40-1.69)$ & 0.588 \\
\hline Parietal lobe, n(\%) & $10(7.52)$ & $9(6.77)$ & $0.95 \square 0.37-2.43 \square$ & 0.922 \\
\hline Temporal lobe, n(\%) & $5(3.76)$ & $10(8.00)$ & $2.23 \square 0.74-6.71 \square$ & 0.146 \\
\hline Occipital lobe, n(\%) & $9(6.77)$ & $6(4.80)$ & $0.70 \square 0.24-2.01 \square$ & 0.500 \\
\hline
\end{tabular}

BMI:Body Mass Index .SD: standard deviation

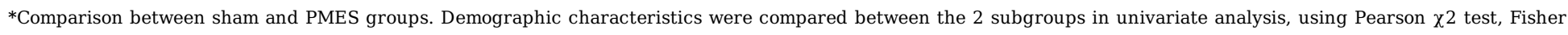

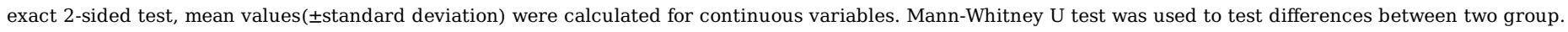

Table 2a The mean value of the MoCA Score and HRSD at 6 months in Sham and PMES groups

\begin{tabular}{llll}
\hline & Sham group(133) & PMES group(125) & P* \\
\hline MoCA Score, (Mean SD) & $27.26 \pm 2.20$ & $28.26 \pm 1.95$ & $<0.001$ \\
HRSD Score, (Mean SD) & $11.54 \pm 4.21$ & $9.58 \pm 3.45$ & $<0.001$ \\
\hline
\end{tabular}

Table $2 b$ The percentage of treatment response and depression remission in Sham and PMES groups

\begin{tabular}{lllll}
\hline & Sham group(133) & PMES group(125) & OR(95\%CI) & P* \\
\hline Treatment response, $\mathrm{n}(\%)$ & $55(41.35 \%)$ & $72(57.60 \%)$ & $1.93(1.18-3.16)$ & 0.009 \\
Depression remission, $\mathrm{n}(\%)$ & $39(29.32 \%)$ & $55(44.00 \%)$ & $1.89(1.13-3.17)$ & $\mathbf{0 . 0 1 4}$ \\
\hline
\end{tabular}

Bold indicates P-values less than 0.05

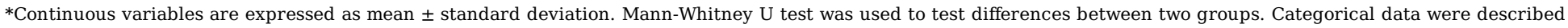
using frequency and percentage, using Pearson $\chi 2$ test, Fisher exact 2-sided test.

Table 3 The mean change in MoCA Score and HRSD in Sham and PMES groups

\begin{tabular}{llll}
\hline & Sham group(133) & PMES group (125) & $\mathrm{P}^{*}$ \\
\hline MoCA Score, (Mean SD) & $2.72 \pm 2.52$ & $3.50 \pm 2.55$ & $\mathbf{0 . 0 0 5}$ \\
HRSD Score, (Mean SD) & $-10.48 \pm 6.10$ & $-11.93 \pm 5.32$ & $\mathbf{0 . 0 3 6}$ \\
\hline
\end{tabular}

Bold indicates P-values less than 0.05

${ }^{*}$ Continuous variables are expressed as mean \pm standard deviation. Mann-Whitney U test was used to test differences between two groups.

\section{Figures}



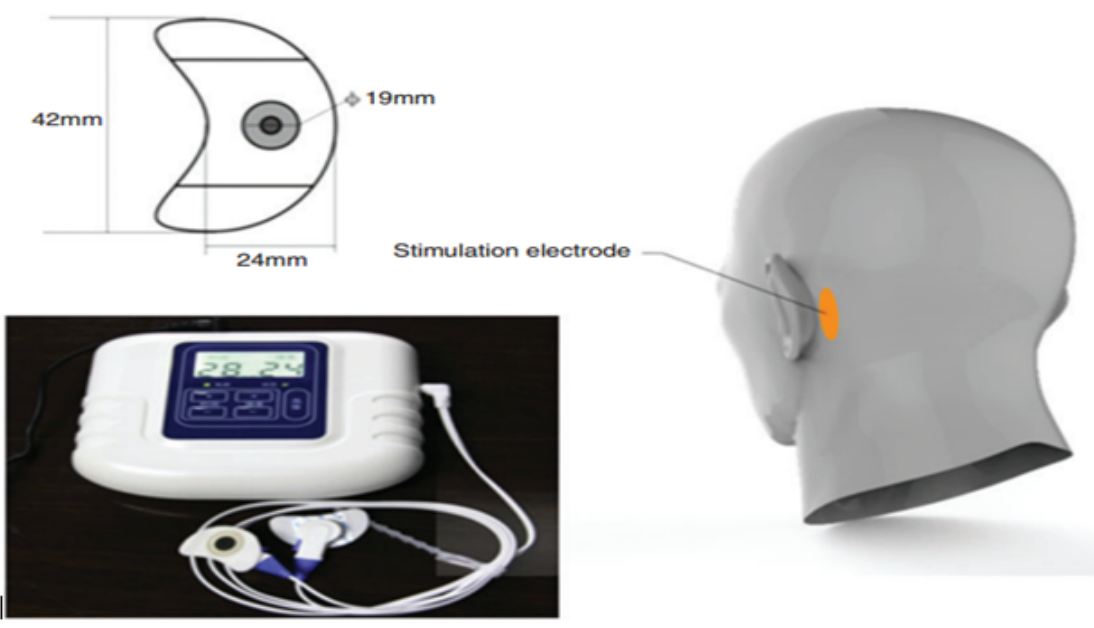

Figure 1

The percutaneous mastoid electrical stimulator (PMES) device and stimulation electrode placed on mastoid area behind the ear

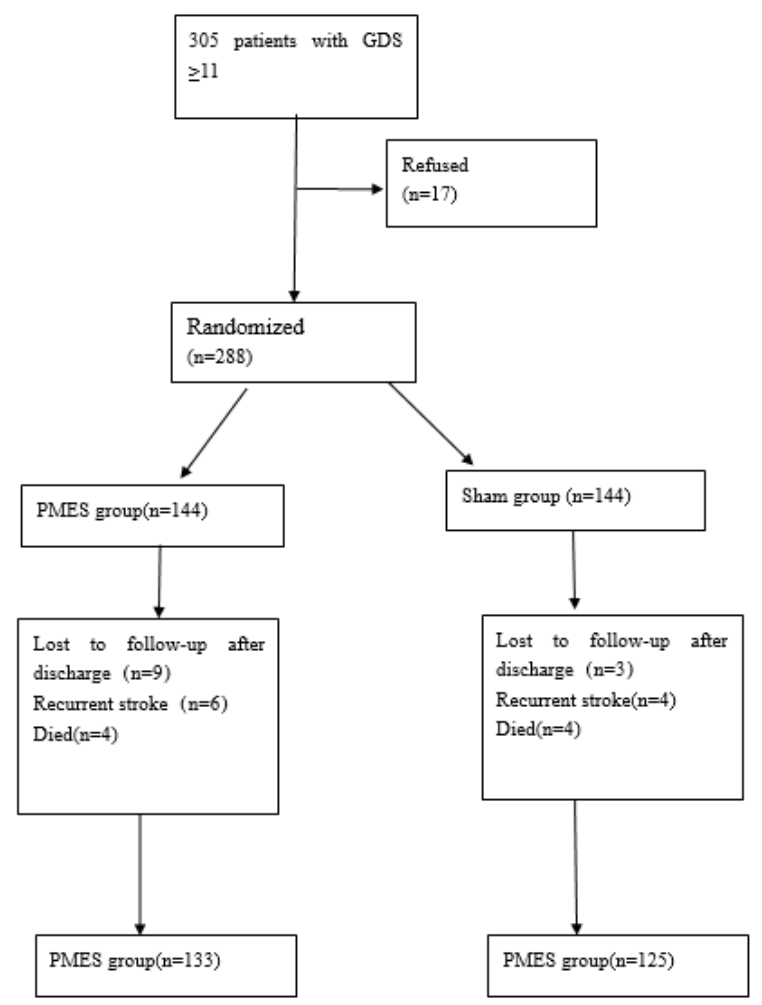

\section{Figure 2}

Patient's flowchart 